\title{
Adiponectin inhibits the generation of reactive oxygen species induced by high glucose and promotes endothelial NO synthase formation in human mesangial cells
}

\author{
FANG YUAN, YI-NING LI, YING-HONG LIU, BIN YI, JUN-WEI TIAN and FU-YOU LIU
}

Department of Nephrology, The Second Xiangya Hospital, Central South University, Changsha, Hunan 410011, P.R. China

Received February 11, 2012; Accepted May 17, 2012

DOI: $10.3892 / \mathrm{mmr} .2012 .931$

\begin{abstract}
The aim of this study was to investigate the effects of adiponectin (ADPN) on high glucose (HG)-induced reactive oxygen species (ROS) and the formation of endothelial nitric oxide (NO) synthase (eNOS) in human glomerular mesangial cells (HMCs), as well as to determine which signaling pathways are modulated by ADPN and the mechanisms involved. HMCs cultured in vitro were randomly divided into 4 groups: the control, $\mathrm{HG}, \mathrm{HG}+$ globular adiponectin $(\mathrm{gAd})$ and $\mathrm{HG}+$ gAd + adenine arabinoside (AraA). The generation of ROS was detected using a fluorescent probe. The mRNA expression and protein levels of eNOS were measured by RT-PCR and western blot analysis, respectively. The phosphorylation of AMP-activated protein kinase (AMPK) was assessed by western blot analysis. HMCs treated with ADPN revealed the time-dependent phosphorylation of AMPK. The treatment of HMCs with HG resulted in increased release of ROS and decreased expression of eNOS compared to the control group $(\mathrm{p}<0.05)$. Cells treated with ADPN showed a decrease in HG-induced ROS $(\mathrm{p}<0.05)$ and an upregulated eNOS expression. The effects of gAd were partly blocked by the AMPK inhibitor, AraA. The results from the present study show that ADPN inhibits the generation of HG-induced ROS in HMCs; it also stimulates eNOS activity, which has a protective effect. The mechanism partly occurs through the stimulation of the AMPK signaling pathway.
\end{abstract}

\section{Introduction}

The incidence of diabetes mellitus is increasing annually. Diabetic nephropathy (DN) is one of the most common and serious chronic complications of diabetes mellitus, and is one

Correspondence to: Dr Yi-Ning Li, Department of General Surgery, The Second Xiangya Hospital, Central South University, No. 139 Renmin Road, Changsha, Hunan 410011, P.R. China

E-mail: yininglicn@163.com

Key words: adiponectin, reactive oxygen species, endothelial nitric oxide synthase, AMP-activated protein kinase, diabetic nephropathy, mesangial cells of the main causes of chronic renal failure. The main pathological changes in DN include hypertrophy and thickening of the glomerular basilemma and renal tubules, extracellular matrix (ECM) accumulation in the mesangium and glomerulosclerosis. The pathogenesis is complicated and is related to many factors, such as glomerular hemodynamic changes, disturbance of biochemical metabolism, oxidative stress (OS), cell factors and genetic predisposition. Compared to other kidney diseases, DN has a poor prognosis and is more difficult to treat, the reasons for which have not yet been fully understood. Increasing evidence has shown that OS plays an important role in DN (1). Kidney cells produce a mass of reactive oxygen species (ROS) and the decrease in anti-oxidants leads to OS. In addition, ROS directly attack unsaturated fatty acids in the body to increase vascular permeability (2), which is closely related to DN, destroy cell membranes in kidney tissues, activate protein kinase and mitogen-activated protein kinase, initiate cytokines and transcription of growth factors, promote proliferation and hypertrophy of cells in kidney tissue and increase ECM synthesis. They also decrease ECM degradation and indirectly injure kidney tissues, resulting in the pathogenic mechanisms of DN.

Adiponectin (ADPN), also known as apM1, Acrp30, GBP28 or AdipoQ, is a recently discovered specific protein secreted by adipose cells; it is abundant in the blood circulation and makes up approximately $0.01 \%$ of the total plasma protein. In addition, ADPN is the most abundant adipose tissue-secreted protein in human plasma. Many studies have shown that ADPN is anti-inflammatory, improves insulin resistance, is anti-atherosclerotic and decreases blood sugar and blood fat levels (3). Previous studies have shown that ADPN has anti-oxidant effects and that its concentration in the blood plasma is closely related to DN. Early studies investigating DN have shown that circulatory ADPN concentration is negatively correlated with urine protein, indicating that impaired endothelial function is related to low ADPN concentration in early DN (4). ADPN concentrations in the urine and blood of patients with progressive-stage DN increase $(5,6)$ (possibly due to the anti-inflammatory and anti-atherosclerotic effects) to abate renal impairment and prevent the adjustment of positive and negative feedback in DN progression (7). Therefore, the present study aimed to investigate the effects of ADPN on OS in human glomerular mesangial cells (HMCs) through the induction of high glucose (HG) levels. The main objective of 
the present study was to investigate the protective effects of ADPN on DN and to determine which signaling pathway is involved in order to clarify the mechanism of its inhibition of diabetic microangiopathy.

\section{Materials and methods}

HMC culture. The HMCs preserved in our research institute were provided by Professor Mei Changlin of the Second Military Medical University of the Chinese People's Liberation Army. The HMCs were cultured at $37^{\circ} \mathrm{C}$ using RPMI-1640 culture medium (Gibco) containing $10 \%$ fetal cattle serum in a $5 \% \mathrm{CO}_{2}$ incubator. The cells were digested and subcultured with $0.25 \%$ pancreatin 2-3 days later. When the cells grew to $85 \%$ fusion, the HMCs were inoculated into a 6-pore plate with a density of $1 \times 10^{6}$ cells $/ \mathrm{ml}$ and cultured in the logarithmic growth phase after $24 \mathrm{~h}$. The culture medium was then changed to serum-free medium and used $12 \mathrm{~h}$ later in a following experiment.

Experimental grouping. Four groups of HMCs were divided randomly into 5 wells/group. The groups were as follows: control group, $\mathrm{HG}$ group, $\mathrm{HG}+$ globular ADPN group ( $\mathrm{HG}+\mathrm{gAd}$ group) and $\mathrm{HG}+\mathrm{gAd}+$ adenine arabinoside group ( $\mathrm{HG}+\mathrm{gAd}+$ AraA group). A 30-mM concentration of culture fluid containing D-glucose was applied to the HG group. The $\mathrm{HG}+\mathrm{gAd}$ group was exposed to $10 \mu \mathrm{g} / \mathrm{ml} \mathrm{gAd}$ (R\&D Co.) for $30 \mathrm{~min}$, to which a $30-\mathrm{mM}$ concentration of D-glucose was added. The $\mathrm{HG}+\mathrm{gAd}+$ AraA group (AraA, an inhibitor of AMP-activated protein kinase (AMPK), was purchased from Sigma Co.) received $\mathrm{HG}$ and $\mathrm{gAd}$ after $30 \mathrm{~min}$ of pre-treatment with $2 \mathrm{mmol} / \mathrm{l}$ AraA.

Determination of ROS levels. Following 24-h culture of the cells in all groups, the orthotropic loading probe was applied according to the manufacturer's instructions. After removing the culture fluid, $1 \mathrm{ml}$ diluted DCFH-DA solution was added into each well to produce a $10-\mu \mathrm{M}$ terminal concentration. The RPMI-1640 serum-free culture medium was used to wash the cells three times after incubation at $37^{\circ} \mathrm{C}$ for $20 \mathrm{~min}$. This was performed to completely remove the probes that were unable to enter the cells. Using a fluorescence spectrophotometer, the cells were collected to determine the fluorescence intensity (FI). The excitation and emission wavelength were 488 and $525 \mathrm{~nm}$, respectively.

Determination of the transcription and protein expression of endothelial nitric oxide (NO) synthase (eNOS). Cell culture continued for $16 \mathrm{~h}$, after which the cells were collected. TRIzol reagent was used to extract the total RNA, after which $1 \mu \mathrm{g}$ total RNA was removed using an RT-PCR kit. This was carried out to synthesize cDNA and conduct PCR, while amplifying eNOS and the reference gene, $\beta$-actin, at the same time. The eNOS properties were as follows: forward primer, 5'-GAGTCCTCACCGCCTTCTC-3'; reverse primer, 5'-AGGAAGCGGGTGGCAGTA-3'; PCR products, 626 bp. The $\beta$-actin properties were as follows: forward primer, 5'-CACCCTGAAGTACCCCATCG-3'; reverse primer, 5'-TTGCCAATGGTGATGACCTG-3'; PCR products, 561 bp. The amplification conditions were as follows: the annealing temperature of eNOS was $54^{\circ} \mathrm{C}$ and that of $\beta$-actin was $58^{\circ} \mathrm{C}$ at
35 cycles. The PCR products were subjected to electrophoresis using a 1.2\% agarose gel and then photographed following ethidium bromide staining. The PCR products were sequenced using a 3730 sequencer.

For western blot analysis, $40 \mu \mathrm{g}$ of total cellular protein were extracted and separated using $8 \%$ SDS-PAGE gel electrophoresis; the protein was also hybridized with polyclonal eNOS antibody after being transferred onto a nitrocellulose membrane. To detect the protein expression of eNOS, coloration was performed using the ECL method and exposure to Kodak film. Quantitative analysis was conducted using UVP. The experiment was repeated 4 times, and the results are expressed as arbitrary units (AU) $(\mathrm{AU}=$ target protein $/ \beta$-actin $\mathrm{x} 100 \%$ ).

Analysis of AMPK expression/phosphorylation. The HMCs in the RPMI-1640 culture medium, which contained $10 \%$ fetal cattle serum, were inoculated into a 6-pore plate. To culture the cells to $85 \%$ fusion, the culture medium was changed to serum-free medium and then $10 \mu \mathrm{g} / \mathrm{ml}$ recombinant human ADPN were added. Following cell activation for 0, 5, 10, 15 and $30 \mathrm{~min}$, the cytoplasmic protein was collected to detect the AMPK expression/phosphorylation (the phospho-AMPK $\alpha$ rabbit polyclonal antibodies were purchased from Cell Signaling Co.).

Statistical analysis. The SPSS11.0 software was used, and the experimental results are expressed as the means \pm SD. Comparisons between the groups were performed using one-way analysis of variance and the t-test. $\mathrm{P}<0.05$ indicated a statistically significant difference.

\section{Results}

Effects of ADPN on the production of ROS. DCFH-DA is lipotropic and freely diffuses into cells and is hydrolyzed as hydrophilic DCFH by esterase in order to remain in the neoplasm. DCFH is easily oxidized as epoptic 2',7'-dichlorofluorescein (DCF) by peroxide (mainly hydrogen peroxide) in the neoplasm. The amount of DCF is directly related to the peroxide in the neoplasm. The experimental results revealed that after $24 \mathrm{~h}$ under the influence of a high concentration of glucose, the FI of the HMCs was significantly enhanced compared to that of the control group $(\mathrm{p}<0.05)$, proving that HG levels stimulated the HMCs to produce ROS. Compared to the HG group, the fluorescence of the $\mathrm{HG}+\mathrm{gAd}$ group was significantly weakened $(\mathrm{p}<0.05)$, indicating that gAd inhibited the production of ROS. When AraA was added, the effects of the inhibition of ADPN on ROS were weakened; no significant difference was found compared to the HG group ( $p>0.05)$. Thus, ADPN inhibited the production of ROS under the stimulation of $\mathrm{HG}$, and this effect was partly inhibited by AraA (Figs. 1 and 2).

Effects of ADPN on the production of eNOS. RT-PCR and western blot analysis results revealed that, after $16 \mathrm{~h}$ of stimulation with $30 \mathrm{mM}$ of D-glucose, the expression of eNOS and mRNA was downregulated by $80.2 \%$ and the protein expression was decreased by $35.3 \%(\mathrm{p}<0.05)$ compared to the control group. The expression of eNOS and mRNA in the $\mathrm{HG}+\mathrm{gAd}$ 

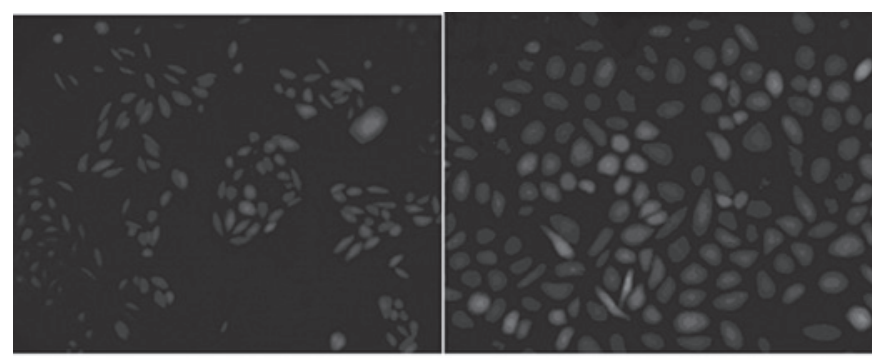

A

B

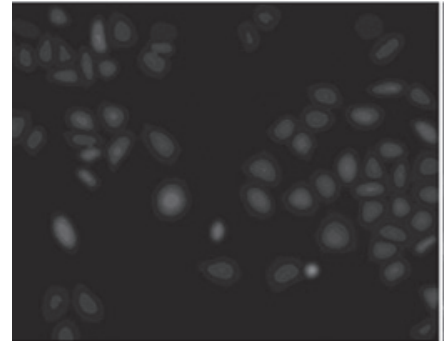

C

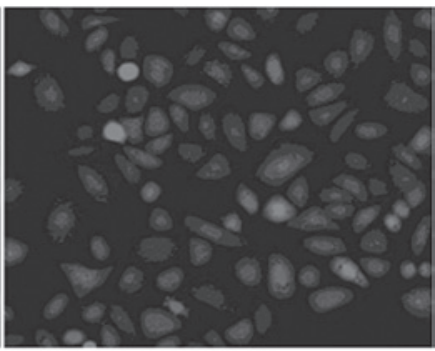

D

Figure 1. Changes in ROS detected by fluorescent probe. (A) Control group. (B) $\mathrm{HG}$ group. (C) $\mathrm{HG}+$ gAd group. (D) $\mathrm{HG}+$ gAd + AraA group. ROS, reactive oxygen species; $\mathrm{HG}$, high glucose; gAd, globular adiponectin; AraA, adenine arabinoside.

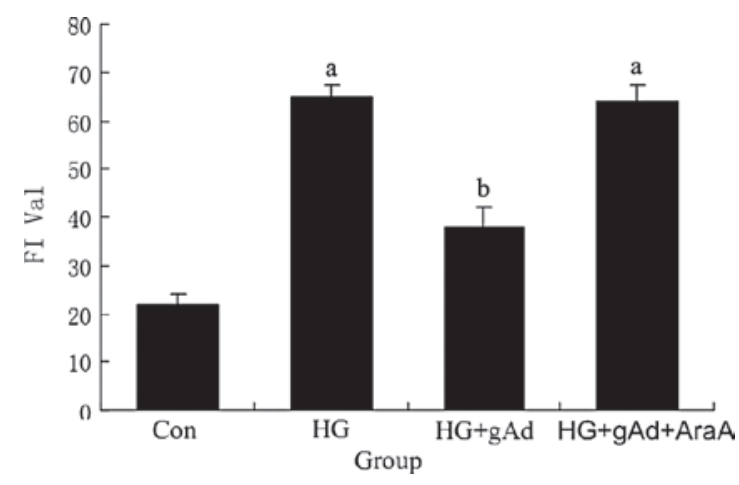

Figure 2. Effects of gAd on the production of ROS in human glomerular mesangial cells (HMCs) compared to the control group ( $\left.{ }^{\mathrm{a}} \mathrm{p}<0.05\right)$ and compared to the HG group $\left({ }^{b} \mathrm{p}<0.05\right)$. gAd, globular adiponectin; ROS, reactive oxygen species; HG, high glucose; AraA, adenine arabinoside.

group was upregulated by $50.5 \%$ and that of protein increased by $30.2 \%(\mathrm{p}<0.05)$ compared to the HG group. Moreover, after AraA was added, the upregulation of eNOS and mRNA decreased; compared to the HG group, no significant difference was found, indicating that ADPN promoted the production of eNOS and subsequently protected the endothelium (Figs. 3 and 4).

Effects of ADPN on AMPK expression/phosphorylation. ADPN stimulated the AMPK expression/phosphorylationin HMCs in a time-dependent manner. The UVP quantitative analysis revealed that without the stimulation of ADPN, the AU value was $0.10 \mu \mathrm{g} / \mathrm{ml}$. In addition, after the stimulation of recombinant human ADPN for 5, 10, 15 and $30 \mathrm{~min}$, the AU values were $06.8 \pm 50.9,487.9 \pm 92.2,943.6 \pm 103.9$ and $399.8 \pm 62.2$, respectively, peaking $15 \mathrm{~min}$ after the stimulation (Fig. 5). The stimulatory effect of ADPN on the AMPK
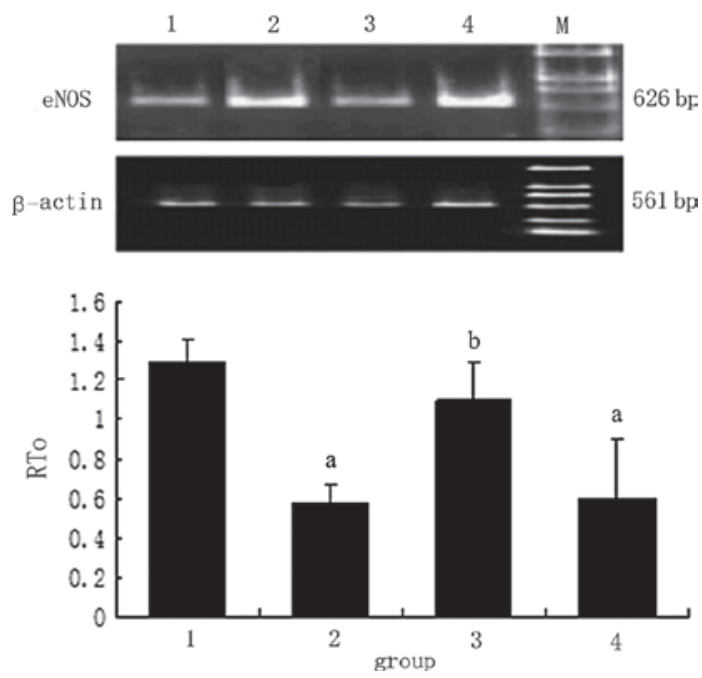

Figure 3. Human glomerular mesangial cell (HMC) eNOS mRNA expression detected by RT-PCR. Lane 1, control group; lane 2, HG group; lane 3 , $\mathrm{HG}+$ gAd group; and lane 4, HG + gAd + AraA group. M, DL2000 marker, compared to the control group $\left({ }^{\mathrm{a}} \mathrm{p}<0.05\right)$, and compared to the HG group $\left({ }^{\mathrm{b}} \mathrm{p}<0.05\right)$. eNOS, endothelial nitric oxide synthase; HG, high glucose; gAd, globular adiponectin; AraA, adenine arabinoside.
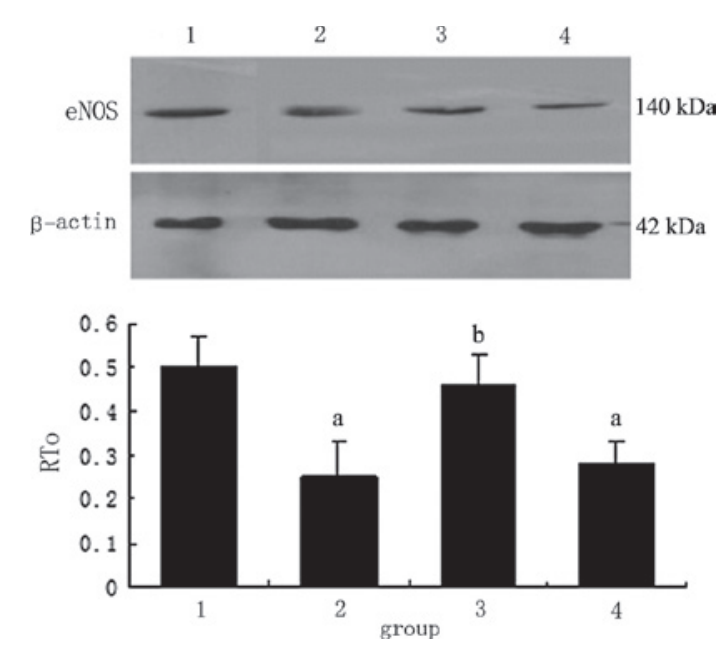

Figure 4. Western blot analysis results of the protein expression in human glomerular mesangial cells and eNOS. Lane 1, control group; lane 2, HG group; lane 3, HG + gAd group; and lane 4, HG + gAd + AraA group, compared to the control group $\left({ }^{\mathrm{a}} \mathrm{p}<0.05\right.$ and $\left.{ }^{\mathrm{b}} \mathrm{p}<0.05\right)$. eNOS, endothelial nitric oxide synthase; HG, high glucose; gAd, globular adiponectin; AraA, adenine arabinoside.

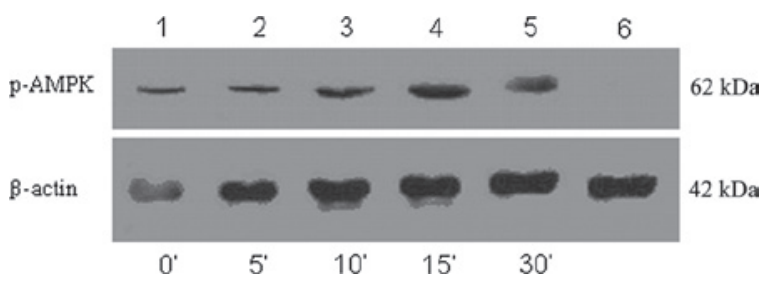

Figure 5. AMP-activated protein kinase (AMPK) expression/phosphorylation stimulated by ADPN at different times. Lane 1, no ADPN; lanes 2-5, treatment with ADPN for 5, 10, 15 and 30 min, respectively; and lane 6, treatment with $2 \mathrm{mM}$ AraA added prior to the stimulation of ADPN. ADPN, adiponectin; AraA, adenine arabinoside.

expression/phosphorylation was completely inhibited by pre-treatment with AraA (2 mM) for $30 \mathrm{~min}$. 


\section{Discussion}

DN is a severe microangiopathy complication of diabetes mellitus, and many studies have investigated its pathogenesis. Multiple factors are attributed to DN and, among these, hyperglycemia is considered an important initiating factor. Nishikawa et al (8) reported that hyperglycemia induces the increase of ROS from the respiratory chain. In a diabetes mellitus state, (nicotinamide adenine dinucleotide phosphate NADPH) oxidase in the kidney tissue is activated by the stimulations of many extracellular signals to mediate ROS generation $(9,10)$. The study by Xu et al (11) showed that in the kidney tissues of diabetic rats, hyperglycemia decreased the production of NADPH, lowered the intracellular glutathione (GSH) levels and weakened the anti-oxidation of kidney tissue through the activation of the protein kinase $\mathrm{A}$, which in turn, reduced the activity of glucose 6-phosphate dehydrogenase (G6PD) produced by NADPH. Ha et al (12) proved that in mesangial and renal tubular epithelial cells, hyperglycemia induces DCF-sensitive ROS in a time-dependent manner. In accordance with the above results, the present study found that the production of ROS in HMCs increased and that the mesangial cells served as an important glomerular source of ROS. Thus, under the conditions of diabetes mellitus, the OS state resultes from the increase in ROS production or the decrease in the clearing of ROS, which by itself attacks the lipids, proteins and DNA to damage the kidney. Previous animal experimental research has indicated that treatment with anti-oxidants reduces proteinuria, abates pathological damage of the kidney and effectively delays the formation and development of DN $(13,14)$.

ADPN is a type of specific protein secreted by adipose cells; it is abundant in circulated blood and is excreted though urine. Several studies have shown that ADPN has an antiinflammatory effect, improves insulin resistance and possesses anti-atherosclerotic and anti-oxidative capabilities. It also inhibits the stimulation of oxidized low-density lipoprotein for the production of ROS in endothelial cells (15), increases the activity of eNOS and protects the blood vessel endothelium from injury by TNF- $\alpha$ (16). The results from the present study prove that employing ADPN to stimulate mesangial cells in vitro inhibits the stimulatory effect of HG on the production of ROS. $\mathrm{NO}$ is the active substance in the blood vessels released from endothelial cells. In the human body, NOS catalyzes 1-arginine to produce NO. eNOS is one of the main rate-limiting enzymes, whose activity is positively correlated with the amount of $\mathrm{NO}$ produced. eNOS continually produces a small amount of NO to relax the smooth muscle; it also inhibits the proliferation of endothelial cells, platelet aggregation and the production of ROS, thereby decreasing OS of the endothelial cells.

In the later stages of DN, the production of eNOS decreases and affects the normal function of NO, thus increasing the production of superoxide (17). In the present study, we found that the HMCs produced eNOS. During the stimulation of HG, the production of eNOS clearly decreased; however, the expression of eNOS and protein secretion evidently increased with the pre-addition of ADPN, thereby protecting the cells. The mechanism was possibly related to the inhibition of the production of ROS in the cells brought about by ADPN. At present, the function of ROS in signal transduction has received increasing attention from investigators. ROS increase the production of ECM, injure DNA of the endothelial cells and regulate the expression of apoptotic genes (18) by activating the signal transduction cascade waterfall and transcription factor and by upregulating the gene expression of transforming growth factor (TGF)- $\beta 1$, angiotensin II (Ang II), monocyte chemoattractant protein-1 (MCP-1) and plasminogen activator inhibitor type 1 (PAI-1). In our study, we found that ADPN upregulated the expression of eNOS by inhibiting the production of ROS; however, the function disappeared when a AMPK blocker was added.

AMPK is a type of protein kinase existing in the tissues of most mammals; it perceives changes in the state of cellular energy metabolism and maintains the balance of the supply and demand of cellular energy by influencing many links to cellular material metabolism. Many factors activate AMPK in mammalian cells, and these factors, such as hypoxia, ischemia and lack of glucose, mainly function by increasing the consumption of cellular adenosine triphosphate (ATP) and inhibiting the production of ATP. Yamauchi et al (19) reported that the mechanism of the signal transduction of ADPN involves the activation of AMPK. In skeletal muscle and hepatic cells, ADPN activates AMPK to reduce blood sugar. Another study has proven that AMPK possibly mediates the signal transmission of ADPN in endothelial cells (20). Activation of AMPK promotes fatty acid oxidation and net synthesis of ATP. In relation to this, the activation of eNOS provides a potential signal connection for the production of ADPN and NO. AMPK plays a vital role in the downstream signal transduction pathways of ADPN receptors and even in maintaining a steady blood glucose level in an organism. In this study, ADPN inhibited the production of ROS in mesangial cells and promoted the increased AMPK expression/phosphorylation in a timedependent manner. Applying AraA as pre-treatment inhibited the phosphorylation of AMPK, partly inhibited the production of eNOS and weakened the inhibition of ADPN in ROS production during the induction of HG levels. AMPK was also found to be included in the signaling pathways modulated by ADPN. Based on studies on liver and skeletal muscle cells, the anti-oxidation of ADPN on mesangial cells was at least partly realized through the phosphorylation of AMPK.

In conclusion, the present study reveals that ADPN protects HMCs against the production of ROS induced by HG levels through anti-oxidation. Our results may provide another possible mechanism for preventing DN by using ADPN. Further studies on the signaling pathways modulated by ADPN and its functional mechanism in the body would provide new ways to prevent and cure $\mathrm{DN}$.

\section{Acknowledgements}

This study was supported by the project of the Hunan Provincial Health Bureau (B2006-043).

\section{References}

1. Stojiljkovic L and Behnia R: Role of renin angiotensin system inhibitors in cardiovascular and renal protection: a lesson from clinical trials. Curr Pharm Des 13: 1335-1345, 2007.

2. Suzuki S, Hinokio Y, Komatu K, et al: Oxidative damage to mitochondrial DNA and its relationship to diabetic nephropathy. Diabetes Res Clin Pract 45: 161-168, 1999. 
3. Okamoto Y, Kihara S, Ouchi N, et al: Adiponectin reduces atherosclerosis in apolipoprotein E-deficient mice. Circulation 106: 2767-2770, 2002.

4. Yilmaz MI,Saglam M, Qureshi AR, et al: Endothelial dysfunction in type-2 diabetics with early diabetic nephropathy is associated with low circulating adiponectin. Nephrol Dail Transplant 23: 1621-1627, 2008.

5. Saraheimo M, Forsblom C and Fagerudd J: Serum adiponectin is increased in type 1 diabetic patients with nephropathy. Diabetes Care 28: 1410-1414, 2005

6. Schalkwijk CG, Chaturvedi N, Schram MT, et al: Adiponectin is inversely associated with renal function in type 1 diabetic patients. J Clin Endocrinol Metab 91: 129-135, 2006.

7. Fujita H, Morii T, Koshimura J, et al: Possible relationship between adiponectin and renal tubular injury in diabetic nephropathy. Endocr J 53: 745-752, 2006.

8. Nishikawa T,Edelstein D and Du XL: Normalizing mitochondrial superoxide production blocks three pathways of hyperglycaemic damage. Nature 404: 787-790, 2000

9. Stokes KY, Clanton EC, Russell JM, et al: NAD (P) H oxidasederived superoxide mediates hypercholesterolemia-induced leukocyte-endothelial cell adhesion. Circ Res 88: 499-505, 2001

10. Rueckschloss U, Galle J, Holtz J, et al: Induction of $\mathrm{NAD}(\mathrm{P}) \mathrm{H}$ oxidase by oxidized low-density lipoprotein in human endothelial cells: antioxidative potential of hydroxyme thylglutaryl coenzyme A reductase inhibitor therapy. Circulation 104 1767-1772, 2001.

11. Xu Y, Osborne BW and Stanton RC: Diabetes causes inhibition of glucose-6-phosphate dehydrogenase via activation of PKA which contributes to oxidative stress in rat kidney cortex. Am J Physiol Renal Physiol 289: 1040-1047, 2005.
12. Ha H, Yang Y, Lee HB, et al: Mechanisms of reactive oxygen species generetion in LLC2PK1 cells cultured under high glucose. J Am Soc Nephrol 13: 531 A, 2002.

13. Davila-Esqueda ME, Vertiz-Hernandez AA and MartinezMorales F: Comparative analysis of the renoprotective effects of pentoxifylline and vitamin $\mathrm{E}$ on streptozotocin-induced diabetes mellitus. Ren Fail 27: 115-122, 2005.

14. Chen L, Jia RH, Ding GH, et al: Effect of irbesartan on oxidative stress and the activity of protein kinase $\mathrm{C}$ in type 2 diabetic rats. Wu Han Da Xue Xue Bao (Yi Xue Ban) 25: 150-153, 2004.

15. Motoshima H, Wu X, Mahadev K, et al: Adiponectin suppresses proliferation an superoxide generation and enhances eNOS activity in endothelial cells treated with oxidized LDL. Biochem Biophys Res Commun 315: 264-271, 2004.

16. Ge Q, Deng HC and Liu JB: Effect of adiponectin on endothelial function. Chin J Endocrinol Metab 1: 15-18, 2006.

17. Pritchard KA Jr, Groszek L, Smalley DM, et al: Native lowdensity lipoprotein increases endothelial cell nitric oxide synthase generation of superoxide anion. Circ Res 77: 510-518, 1995.

18. Ha $\mathrm{H}$ and Lee HB: Reactive oxygen species amplify glucose signalling in renal cells cultured under high glucose and in diabetic kidney. Nephrology 1: 7-10, 2005.

19. Yamauchi $\mathrm{T}$, Kamon J, Ito $\mathrm{Y}$, et al: Cloning of adiponectin receptors that mediate antidiabetic metabolic effects. Nature 423: 762-769, 2003

20. Chen H, Montagnani M, Funahashi T, et al: Adiponectin stimulates production of nitric oxide in vascular endothelial cells. J Biol Chem 278: 45021-45026, 2003. 